\title{
Análise da influência de dois processos distintos de moagem nas propriedades do pó precursor e do cimento de beta-TCP
}

\section{(Analysis of the influence of two different milling processes on the properties of beta-TCP precursor powder and cement)}

\author{
H. A. I. Cardoso ${ }^{1}$, M. Motisuke ${ }^{2}$, C. A. C. Zavaglia ${ }^{1}$ \\ ${ }^{l}$ Laboratório de Biomecânica, DEMA, FEM, UNICAMP, Cidade Universitária Zeferino Vaz, C.P. 6122 \\ Campinas, SP 13083-970 \\ ${ }^{2}$ Instituto de Ciência e Tecnologia, UNIFESP, R. Talim 330, Vila Nair, S. José dos Campos, SP 12231-280
}

\begin{abstract}
Resumo
São várias as características que tem colocado os cimentos de fosfato de cálcio em evidência na área dos biomateriais, como sua bioatividade e reabsorção in vivo. Neste trabalho, analisou-se a influência de dois processos de moagem nas propriedades morfológicas do pó de [beta]-fosfato tricálcico, [beta]-TCP, e na resistência mecânica do cimento. $\mathrm{O}$ pó foi obtido via reação no estado sólido de $\mathrm{CaCO}_{3}$ e $\mathrm{CaHPO}_{4}$ a $1050{ }^{\circ} \mathrm{C}$, apresentando pureza de fase e ausência de elementos tóxicos. O pó foi moído em: (A) moinho de bolas e (B) moinho vibratório de alta energia; sendo analisado por MEV e distribuição granulométrica. Os pós apresentaram propriedades diferentes com relação à distribuição e tamanho médio de grão. Finalmente, o cimento preparado com o pó submetido ao processo (B) apresentou valores de resistência mecânica significativamente maiores que o preparado com o pó submetido ao processo (A). Conclui-se que o processo de moagem (B) é muito mais eficiente que o processo (A).

Palavras-chave: [beta]-TCP, moagem, tamanho de partícula, cimento ósseo.
\end{abstract}

\begin{abstract}
There are several characteristics that put calcium phosphate cements in evidence, like its bioactivity and in vivo resorption. The influence of two milling processes on the morphological properties of the [beta]-tricalcium phosphate powder, [beta]-TCP, and in the mechanical properties of the cement were analyzed. The powder was obtained by solid state reaction of $\mathrm{CaCO}_{3}$ and $\mathrm{CaHPO}_{4}$ at $1050{ }^{\circ} \mathrm{C}$. It showed high phase purity and absence of toxic elements. The powder was processed in ball mill (A) and high-energy vibratory mill (B), with posterior analysis by SEM and particle size distribution. The powders showed different average and distribution of grain size. Finally, the cement prepared with powder submitted to process $(B)$ showed values of axial tensile strength significantly greater than that prepared with powder submitted to process (A). The milling process $(B)$ is much more efficient than the process $(A)$.
\end{abstract}

Keywords: [beta]-TCP, milling, particle size, bone cement.

\section{INTRODUÇÃO}

Os cimentos de fosfato de cálcio são utilizados cada vez mais como biomateriais por se ligarem química e biologicamente com o tecido ósseo adjacente com posterior reabsorção [1]. Suas aplicações vão desde reconstrução de partes de tecidos ósseos traumatizados [2,3] a formulações que apresentam ações antimicrobianas [4, 5]. A sua principal limitação é a sua baixa resistência mecânica, que inviabiliza sua aplicação em situações de grande solicitação [6]. Dentre as propriedades que influenciam no comportamento mecânico do material, como a porosidade [7] e a cristalinidade [8], destaca-se a distribuição de tamanho de partículas. Em um estudo pioneiro [9], foi feita uma modelagem da hidrólise do cimento de [alfa]-fosfato tricálcico baseado no tamanho de partículas. Observou-se que o mecanismo cinético da reação de hidrólise é controlado inicialmente pela área superficial específica, propriedade relacionada com o tamanho de partículas, e depois pela difusão do líquido através do cimento. Estudos subseqüentes [10] mostraram que a redução do tamanho médio de partícula em cinco vezes, provocou uma diferença não significativa na resistência mecânica final do cimento de [alfa]-TCP, entretanto a aceleração da reação de pega causou um aumento precoce da resistência mecânica do cimento obtido com o pó mais fino. A influência de três diferentes processos de moagem (moinho de bolas, moagem criogênica e moinho planetário) na redução do tamanho de partículas do [alfa]-fosfato tricálcico foi analisada [11]. Foi comprovado que todos os métodos foram eficientes na redução do tamanho de partículas, otimizando 
as propriedades mecânicas do material. Entretanto, períodos prolongados de moagem causaram uma aglomeração no pó, diminuindo a resistência mecânica final dos cimentos, independente da técnica de moagem empregada. Entretanto, estudos que analisam as propriedades do tamanho de partícula e dos processos de moagem no pó de [beta]-TCP, um polimorfo do fosfato tricálcico, são pouco difundidos.

O objetivo deste trabalho foi analisar as influências de dois processos de moagem (moinho de bolas e moinho vibratório de alta energia) nas propriedades do pó e do cimento de [beta]-TCP.

\section{MATERIAIS E MÉTODOS}

O [beta]-TCP foi obtido via reação do estado sólido por meio da mistura de hidrogenofosfato de cálcio, ou monetita $\left(\mathrm{CaHPO}_{4}\right.$, Sigma Aldrich, lote: 1382643), e carbonato de cálcio $\left(\mathrm{CaCO}_{3}\right.$, Synth lote: 102306$)$ em relação estequiométrica conforme a reação descrita em (A):

$$
\mathrm{CaCO}_{3(\mathrm{~S})}+2 \mathrm{CaHPO}_{4(\mathrm{~S})} \rightarrow \mathrm{Ca}_{3}\left(\mathrm{PO}_{4}\right)_{2(\mathrm{~S})}+\mathrm{H}_{2} \mathrm{O}_{(\mathrm{g})}+\mathrm{CO}_{2(\mathrm{~g})}
$$

Após homogeneização os reagentes foram calcinados a $1050^{\circ} \mathrm{C}$ durante $6 \mathrm{~h} \mathrm{com}$ taxa de aquecimento $10^{\circ} \mathrm{C} / \mathrm{min}$. $\mathrm{O}$ pó foi moído a seco por dois processos diferentes, Tabela I:

Tabela I - Parâmetros de moagem: processos A e B. [Table I - Milling parameters: processes A and B.]

\begin{tabular}{ccccc}
\hline Processo & moinho & $\begin{array}{c}\text { bolas / } \\
\text { diâmetro } \\
(\mathrm{mm})\end{array}$ & $\begin{array}{c}\text { tempo } \\
\text { de } \\
\text { moagem }\end{array}$ & $\begin{array}{c}\text { rotação } \\
\text { do motor } \\
(\mathrm{rpm})\end{array}$ \\
\hline A & $\begin{array}{c}\text { Horizontal } \\
\text { de Bolas }\end{array}$ & $\begin{array}{c}\text { Alumina } \\
/ 15\end{array}$ & 2 dias & 40 \\
& $\begin{array}{c}\text { Vibratório } \\
\text { de Alta }\end{array}$ & $\begin{array}{c}\text { Zircônia } \\
\text { B } 6,5\end{array}$ & 4 horas & 50 \\
\hline
\end{tabular}

A eficiência do método de síntese proposto foi avaliada determinando-se as fases cristalinas presentes nos pós de [beta]-TCP por meio da difração de raios X (Rigaku DMAX2200), $2 \theta=20$ a $40^{\circ}, 30 \mathrm{~mA}, 40 \mathrm{kV}, 0,01^{\circ} / \mathrm{s}$. A presença de elementos traço tóxicos nos pós de [beta]TCP foi verificada por uma análise semi-quantitativa de fluorescência de raios X (Rigaku RIX-3100). A distribuição do tamanho de partículas foi determinada por difração a laser (Mastersizer Hydra 2000, Malvern). Finalmente, a morfologia e a homogeneidade do pó foram analisadas por microscopia eletrônica de varredura (JEOL 840A) das amostras recobertas com uma fina camada de ouro (Baltec SCD 050). As amostras foram analisadas de duas formas: a primeira com o pó aglomerado conforme será utilizado na preparação do cimento; na segunda o pó foi disperso em isopropanol (Synth) em ultrassom durante $15 \mathrm{~min}$. Em seguida, uma pequena gota de suspensão foi colocada sobre o porta-amostra.

Após completa caracterização dos pós, os cimentos foram preparados por meio da mistura do pó seco de $\beta$-TCP com uma solução aquosa com concentração $2,0 \mathrm{~mol} / \mathrm{L}$ de $\mathrm{H}_{3} \mathrm{PO}_{4}$ numa relação líquido pó de $0,8 \mathrm{~mL} / \mathrm{g}$. A pasta foi moldada em moldes cilíndricos com $0,6 \mathrm{~mm}$ de diâmetro por $12 \mathrm{~mm}$ de altura. Pouco tempo depois, os cilindros foram desmoldados e secados ao ar, para posterior medição da resistência mecânica. Foram preparados cinco corpos de prova para cada processo.

A resistência mecânica à compressão axial dos cimentos obtidos pelos dois processos foi determinada por meio de um ensaio de compressão uniaxial em uma máquina universal de ensaios (EMIC DL 2000, 0,5 mm/min, célula de carga de $0,5 \mathrm{kN})$. Para analisar a significância estatística da diferença entre as duas amostras foi feito o teste ANOVA.

\section{RESULTADOS E DISCUSSÃO}

A Tabela II apresenta o resultado da fluorescência de raios $\mathrm{X}$ para os elementos minoritários presentes no pó. Sabe-se que mesmo os elementos em maiores quantidades não são prejudiciais à biocompatibilidade e à biofuncionalidade do material, pois estão presentes na fase mineral óssea. Além

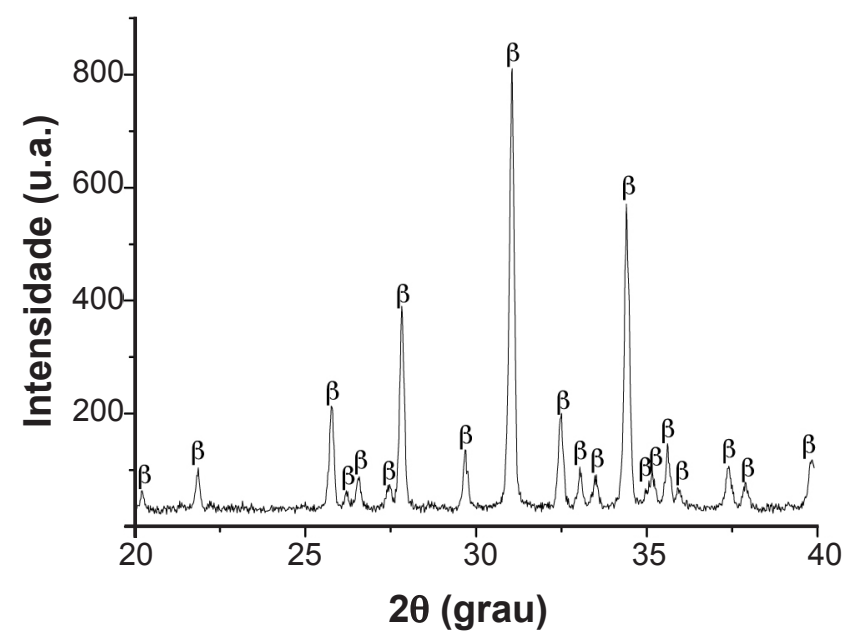

Figura 1: Difratograma de raios $\mathrm{X}$ do pó: picos apenas de $\beta$-TCP (JCPDS 09-0169).

[Figure 1: X-ray diffraction pattern of the powder: only peaks of $\beta$-TCP (JCPDS 09-0169).]

Tabela II - Elementos minoritários presentes no pó de $\beta$-TCP.

[Table II - Trace elements present in $\beta$-TCP powder.]

\begin{tabular}{cccccccccccc}
\hline Elemento & $\mathrm{Cu}$ & $\mathrm{Au}$ & $\mathrm{Ni}$ & $\mathrm{S}$ & $\mathrm{K}$ & $\mathrm{Fe}$ & $\mathrm{Al}$ & $\mathrm{Sr}$ & $\mathrm{Si}$ & $\mathrm{Na}$ & $\mathrm{Mg}$ \\
\hline$\%$ massa & 0,002 & 0,004 & 0,005 & 0,009 & 0,009 & 0,019 & 0,028 & 0,072 & 0,073 & 0,193 & 0,254 \\
\hline
\end{tabular}


disso, o Mg é um conhecido agente estabilizador do $\beta$-TCP.

A Fig. 1 apresenta o difratograma de raios $X$ do pó. Notase que o pó utilizado é constituído apenas da fase de $\beta$-TCP e os elementos traço não interferiram na síntese do pó. Não se verifica a presença de nenhuma outra fase presente, ou mesmo interferências nos picos do difratograma: todos os picos são de $\beta$-TCP.

$\mathrm{Na}$ Fig. 2, por meio da microscopia eletrônica de varredura, o pó é analisado aglomerado, forma em que é aplicado na preparação do cimento.

Por meio dessa análise qualitativa, é possível observar que o pó obtido pelo Processo A apresenta uma distribuição heterogênea, com partículas de formas e tamanhos diversos. O formato pontiagudo das partículas maiores revela que o processo em questão foi pouco eficiente, indicando que o tempo de moagem não foi suficiente para a estabilização do processo ou que a força de atrito e o impacto devido à colisão entre as bolas e o pó não foi suficiente para homogeneizar o pó. Como o tempo de moagem do processo foi de dois dias, dificilmente esse foi o fator limitante na moagem do pó. Provavelmente, o processo não apresenta energia suficiente para obter um pó mais homogêneo. Diferentemente, o pó obtido pelo Processo B apresenta uma distribuição mais homogênea, com partículas menores e formato mais
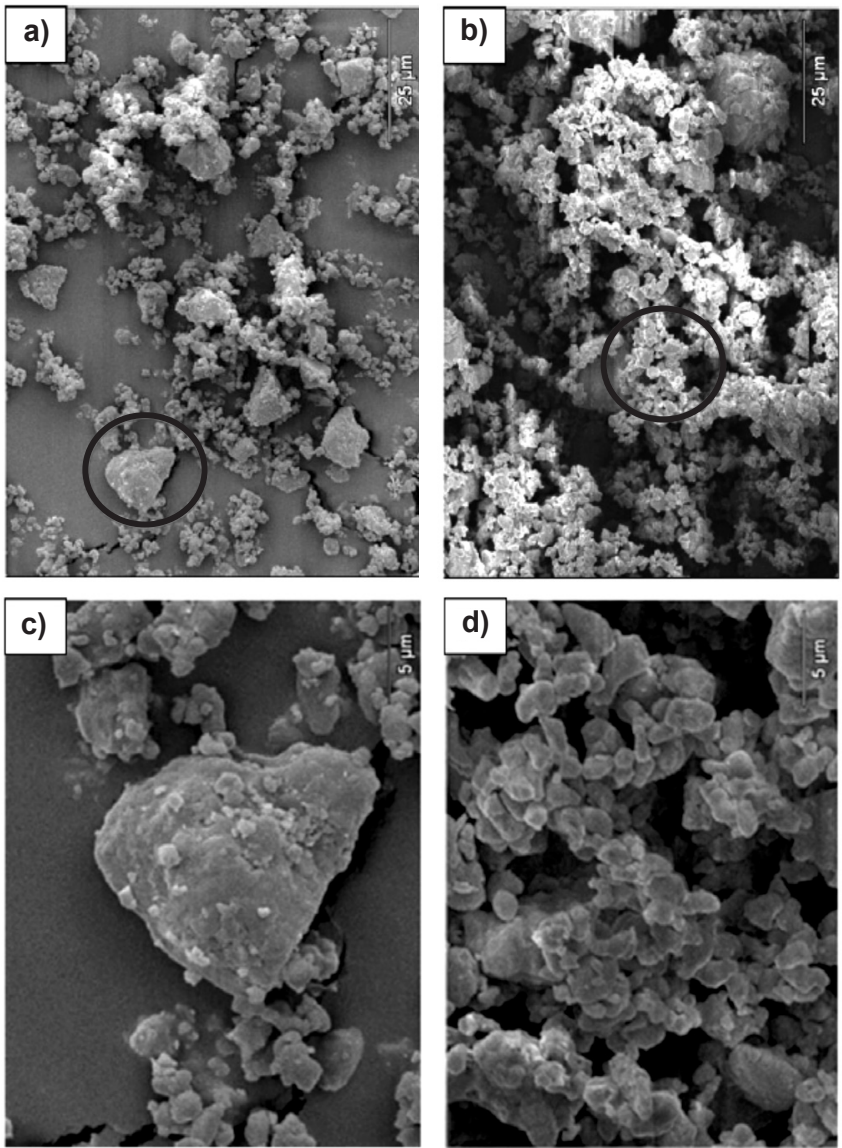

Figura 2: Micrografias de microscopia eletrônica de varredura: Processo A (imagens "a" e "b") e pelo Processo B (imagens "c" e "d"). [Figure 2: Scanning electron microscopy images: Process A (images " $a$ " and " $b$ ") and Process B (images " $c$ " and " $d$ "). ] uniforme. A energia envolvida, neste caso, foi eficiente para uniformizar o formato e a distribuição de tamanho de partícula, apesar do tempo relativamente baixo de moagem.

Para melhor análise das características do pó, foi feita análise de microscopia eletrônica de varredura das partículas também desaglomeradas, conforme se observa na Fig. 3.
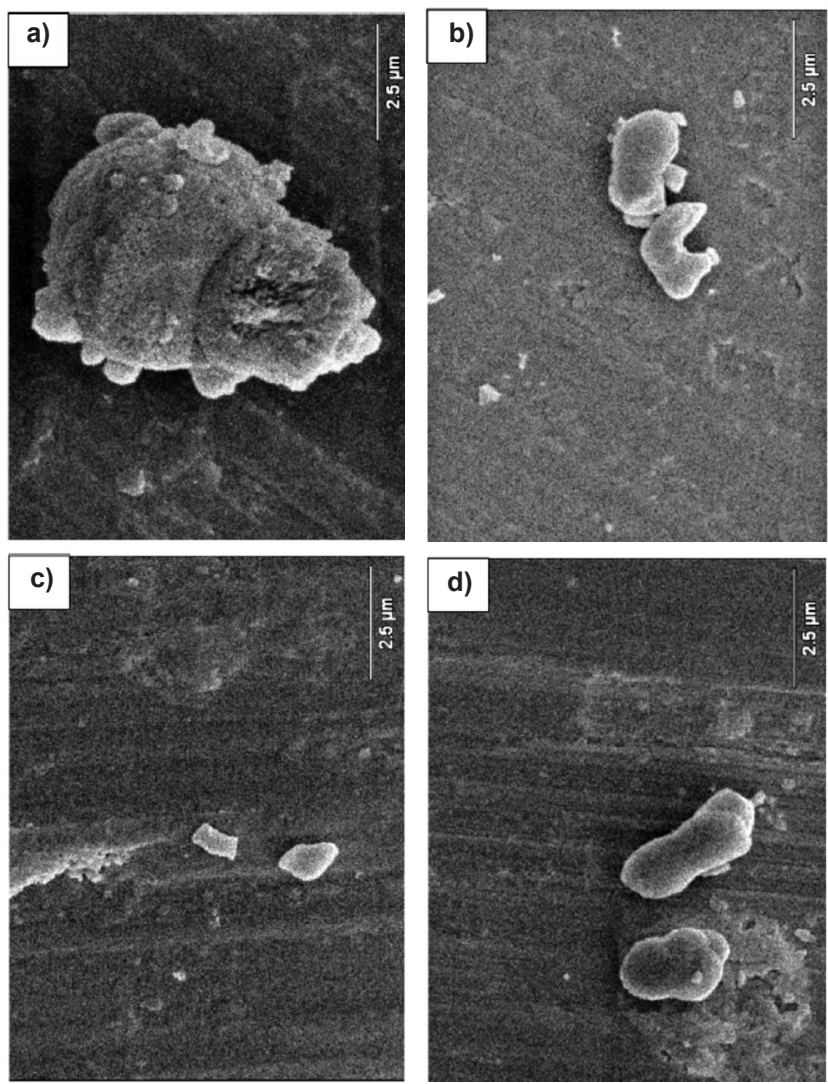

Figura 3: Micrografias de microscopia eletrônica de varredura com pó disperso: Processo A ("a" e "b) e Processo B ("c" e "d").

[Figure 3: Scanning electron microscopy micrographs of dispersed powder: Process A (images " $a$ " and " $b$ ") and Process $B$ (images "c" and " $d$ ").]

É possível confirmar que o pó obtido pelo Processo A apresenta partículas de formas extremamente variadas, diferentemente do apresentado pelo Processo B. De uma forma geral, observa-se que o pó obtido pelo Processo B apresenta partículas menores. No entanto, essa análise é qualitativa. Assim, espera-se que a análise granulométrica confirme quantitativamente essa diferença. A distribuição granulométrica obtida para os processos A e B estão apresentadas na Fig. 4.

A análise dos diagramas permite afirmar que a distribuição de tamanho de partícula dos dois processos é realmente diferente. O Processo A apresenta uma distribuição bimodal, já demonstrando o comportamento heterogêneo na distribuição do material, enquanto que o processo B apresenta uma distribuição mais aproximada do formato de uma gaussiana. Apesar da faixa de distribuição ser próxima, entre 0,3 e $40 \mu \mathrm{m}$, é notável que o Processo A apresenta uma concentração mais dispersa e o Processo B 


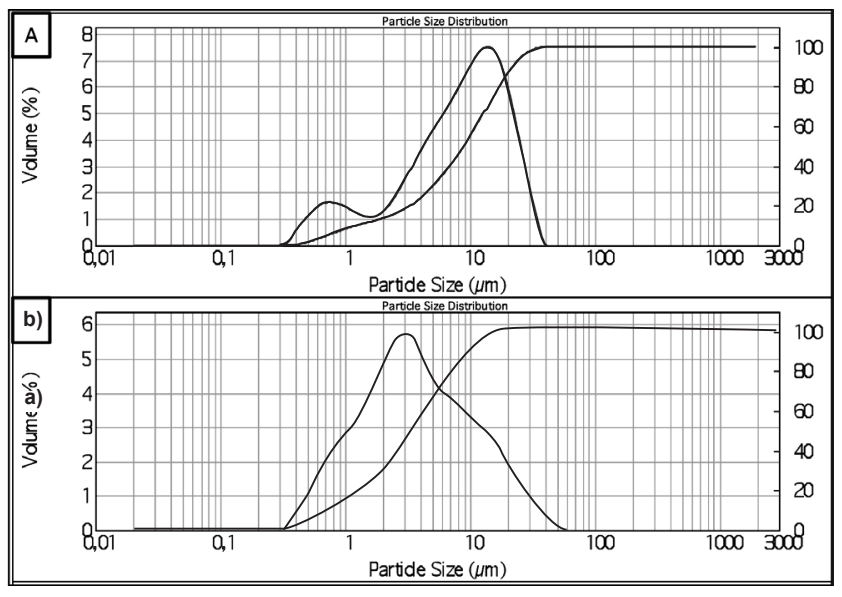

Figura 4: Distribuição granulométrica: Processos A e B. [Figure 4: Size distribution: Processes A and B.]

uma distribuição mais concentrada, mais clara por meio da análise da curva que representa o volume acumulado. Além disso, o tamanho médio de partícula do Processo A foi de aproximadamente $8,5 \mu \mathrm{m}$, enquanto que o do Processo B foi de $3,5 \mu \mathrm{m}$, reiterando a maior eficiência do Processo B em relação ao A.

Na Fig. 5 pode-se observar a distribuição da resistência mecânica por meio do Boxplot dos valores obtidos no ensaio.

É notável a diferença de resistência entre os dois cimentos. O cimento obtido pelo Processo A apresenta uma faixa de distribuição muito maior que o obtido pelo Processo B, indicando, inclusive, uma falta de reprodutibilidade nos resultados. O Processo B apresenta uma faixa de distribuição estreita e mais confiável. Além disso, o valor médio obtido no Processo A $(0,68 \pm 0,53 \mathrm{MPa})$ foi menos da metade do obtido pelo Processo B $(1,65 \pm 0,20 \mathrm{MPa})$. A falta de reprodutibilidade é confirmada pelo desvio padrão referente ao Processo A, próximo do valor absoluto da resistência mecânica encontrada, indicando uma dispersão muito grande dos resultados. A distribuição mais homogênea do tamanho de partículas garantiu uma reação de pega mais uniforme em todo o cimento, garantindo melhores propriedades mecânicas.

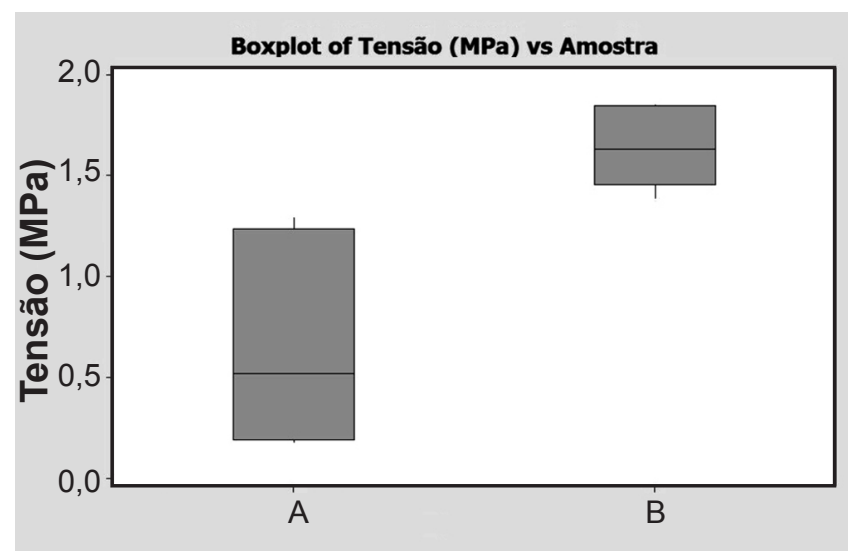

Figura 5: Boxplot da distribuição da resistência mecânica à compressão axial.

[Figure 5: Boxplot distribution of the compressive axial strength.]
Além do tamanho menor de partícula acelerar a reação de pega e auxiliar na aquisição de resistência mecânica.

Finalmente, a análise estatística pelo método ANOVA com $95 \%$ de confiabilidade indicou diferença estatística entre as resistências obtidas pelos dois cimentos $(\mathrm{p}=0,005)$.

\section{CONCLUSÕES}

Foi possível obter $\beta$-TCP elevada pureza de fase cristalina. $\mathrm{O}$ processo de moagem em moinho de bolas horizontal não foi eficiente, apesar do tempo de moagem prolongado, apresentando partículas com formatos diversos e uma distribuição heterogênea do tamanho de partículas. Já o processo de moagem com moinho vibratório de alta energia foi eficiente, mesmo com o tempo curto de moagem, apresentando partículas com formatos e distribuição mais homogênea. O cimento obtido do pó moído via moinho vibratório de alta energia apresentou resistência mecânica média maior que o dobro do obtido pelo moinho horizontal, além de uma faixa de distribuição muito mais estreita e confiável.

\section{AGRADECIMENTOS}

Os autores agradecem ao Conselho Nacional de Desenvolvimento Científico e Tecnológico $(\mathrm{CNPq})$ e à Fundação de Amparo à Pesquisa do Estado de S. Paulo (Fapesp) pelos recursos disponibilizados durante o desenvolvimento desse trabalho. E também a UNIFEI pelo auxílio nos testes de resistência mecânica.

\section{REFERÊNCIAS}

[1] A. J. Ambard, L. Mueninghoff, J. Prosthodont 15 (2006) 321.

[2] S. V. Dorozhkin, Materials 2 (2009) 221.

[3] D. Apelt, F. Theiss, A. O. El-Warrak, K. Zlinszky, R. Bettschart-Wolfisberger, M. Bohner, S. Matter, J. A. Auer, B. von Rechenberg, Biomater. 25 (2004) 1439.

[4] S. Larsson, T. W. Bauer, Clinical Orthopaedics Related Res. 395 (2002) 23.

[5] U. Gbureck, O. Knappe, N. Hofmann, J. E. Barralet, J. Biomedical Mater. Res. Part B: Applied Biomater. 83 (2007) 132.

[6] H. E. Briak, D. Durand, P. Boudeville, J. Mater. Sci.: Mater. Medicine 19 (2008) 737.

[7] J. E. Barralet, T. Gaunt, A. J. Wright, I. R. Gibson, J. C. Knowles, J. Biomedical Mater. Res. 63 (2002) 1.

[8] M. Bohner, T. J. Brunner, W. J. Stark, J. Mater. Chem. 18 (2008) 5669.

[9] M. P. Ginebra, E. Fernández, F. C. M. Driessens, J. A. Planell, J. Am. Ceram. Soc. 82 (1999) 2808.

[10] M. P. Ginebra, F. C. M. Driessens, J. A. Planell, Biomater. 25 (2004) 3453.

[11] V. Jack, F. J. Buchanan, N. J. Dunne, J. Eng. Medicine $222(2008) 19$.

(Rec. 26/05/2011, Rev. 10/07/2011, Ac. 09/08/2011) 